\title{
ELABORACIÓN DE PASTA DE ACEITUNA NEGRA UTILIZANDO EL HIDROCOLOIDE GOMA TRAGACANTO
}

\author{
MAKING BLACK OLIVE PASTE USING HYDROCOLLOID GUM \\ TRAGACANTH
}

\author{
${ }^{1}$ Marcial Alfredo Castillo Cohaila
}

\begin{abstract}
RESUMEN
Se evaluó la calidad sensorial de la pasta de aceituna negra utilizando el hidrocoloide goma tragacanto, determinándose su efecto en la textura, sensación en boca, sinéresis y aspecto general. La textura de la pasta de aceituna negra, no se vio influenciada por el uso del hidrocoloide en el procesamiento. La sensación en boca de la pasta de aceituna negra, no se vio afectada por el uso del hidrocoloide en el procesamiento. La sinéresis y el aspecto general de la pasta de aceituna negra se vio afectada positivamente por el uso del hidrocoloide.
\end{abstract}

Palabras clave: Pasta de aceituna, hidrocoloide.

\section{ABSTRACT}

The Sensory quality of black olive paste was evaluated using the hydrocolloid gum tragacanth. determining its effect on texture, sensation in mouth, and general appearance. The texture of black olive paste was not influenced by the use of hydrocolloids in processing. The feeling in the mouth of black olive paste was not affected by the use of hydrocolloid in processing. Syneresis and general appearance of black olive paste was positively affected by the use of the hydrocolloid.

Keywords: Olive paste, hydrocolloid.

\section{I.INTRODUCCIÓN}

El nombre de hidrocoloide engloba a sustancias naturales poliméricas solubles o dispersables en agua. Por esa razón se les puede considerar como fibras solubles. Aunque en este grupo también se incluye a los almidones y algunas proteínas, como la gelatina, generalmente el término hidrocoloide se aplica a sustancias de composición polisacárida. De forma coloquial se les denomina "gomas".

Como característica principal y común se puede destacar que son moléculas altamente hidrofilicas que actúan sobre el agua que se encuentra libre en el medio donde se aplican, llegando a reducir su movilidad y aumentando así la viscosidad. En este sentido la estructura del polímero (lineal o ramificado, su grado de ramificación, etc.) es de gran importancia ya que de ella depende la capacidad de retención de agua (volumen de agua que pueden llegar a incorporar) y, por tanto, las características reológicas y de textura que impartirá al producto acabado.

Se ha comprobado que algunas mezclas de dos hidrocoloides, sean o no gelificantes, pueden ofrecer fenómenos de sinergia que conducen a comportamientos reológicos diferentes, circunstancia que la industria alimentaria ha sabido aprovechar usando mezclas de carragenina y garrofín o de alginatos y pectinas. (Bello, 2000).
De todos los hidrocoloides, la goma tragacanto es el que imparte mayor viscosidad, dando soluciones coloidales que tienen una textura similar a un gel débil.

La goma tragacanto es soluble en agua fría y es estable al calor y a valores bajos de $\mathrm{pH}$ (por debajo de $\mathrm{pH}=2$ ). Es eficiente añadida en procesos de emulsión, potenciando la mezcla e integración. En condimentos como kétchup, la goma tragacanto funciona como un estabilizador y espesante en la fase acuosa. Suele utilizarse en aliños y salsas porque imparte una textura cremosa. Los "milkshakes" hechos con goma tragacanto pueden tener niveles más bajos de grasa sin sacrificar la viscosidad. También se utiliza en helados para dar cuerpo y textura, conferir cremosidad, espesar y estabilizar. (Cubero N. et. al., 2002)

Para la elaboración de la pasta de aceituna en primer lugar se obtiene la pulpa de las aceitunas, se las somete a molienda lo más finamente posible, se adiciona sorbato de potasio o benzoato de sodio, o ambos para conservar las aceitunas. Para ajustar el $\mathrm{pH}$ se acidifica con ácido láctico. La pasta se envasa y se almacena, según la Figura $\mathrm{N}^{\circ} 0$ 1. (Schwartz et al., 2009).

La pasta de aceitunas es el resultado de moler finamente pulpa de aceituna. Para su conservación pueden incorporarse ingredientes o aditivos. (Hashiguchi, 2005).

El objetivo principal del presente trabajo de investigación fue determinar el efecto de la utilización del 


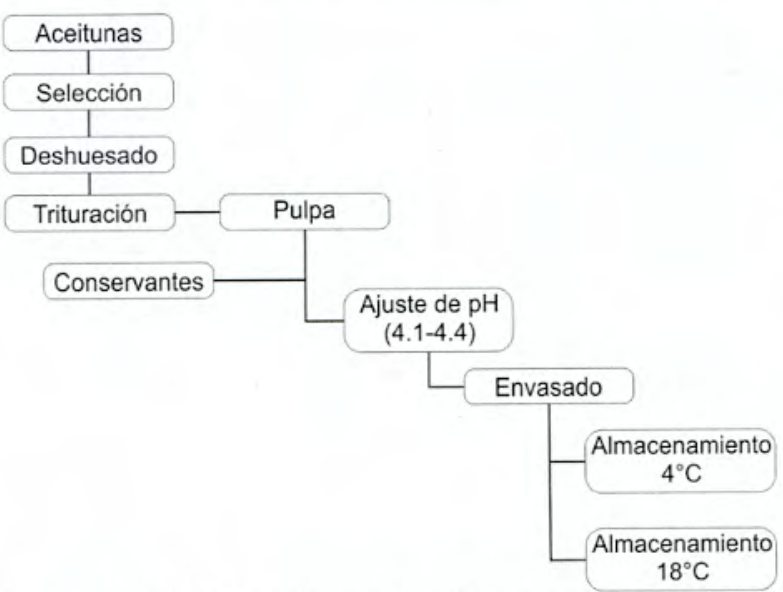

Figura $\mathrm{N}^{\circ} 01$. Operaciones en elaboración de pasta de aceituna

Fuente:(Schwartzet al., 2009)

hidrocoloide goma tragacanto en la calidad de la pasta de aceituna negra.

Los objetivos específicos fueron:

- Determinar la concentración de hidrocoloide goma tragacanto a utilizar.

- Determinar el aporte del hidrocoloide goma tragacanto a la calidad de la pasta de aceituna.

\section{MATERIALES Y MÉTODOS}

\subsection{Material}

- Aceitunas negras variedad Sevillana: 7 kilogramos

- Depósitos para pasta de aceitunas: 13 unidades

- Hidrocoloide: goma tragacanto.

- Material de laboratorio: Balanza gramera.

- Licuadora doméstica Marca Oster, con vaso pequeño.

- 01 computadora Acer Dual Core

- Papelbond

\subsection{Metodologia}

El proyecto se desarrolló por la metodología que se observa en la Figura $\mathrm{N}^{\circ} 02$.

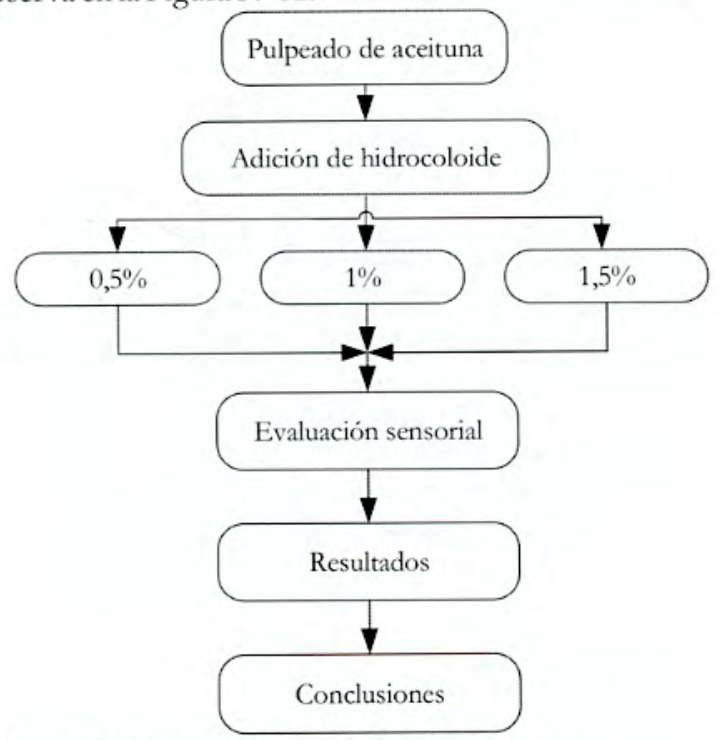

Figura $\mathrm{N}^{\circ}$ 02. Metodología de la investigación

\subsection{Diseño experimental}

El diseño experimental consistió de tres tratamientos con 4 repeticiones, como puede verse en la Tabla $\mathrm{N}^{\circ} 01$, evaluándose transcurridas 2 semanas la calidad sensorial de las muestras.

Tabla No 01 . Diseño experimental

\begin{tabular}{ccccc}
\hline Prueba & \multicolumn{5}{c}{ Adición del hidrocoloide } \\
& $\mathbf{0} \%$ & $\mathbf{0 , 5} \%$ & $\mathbf{1} \%$ & $\mathbf{1 , 5} \%$ \\
\hline 1 & $\mathrm{a}_{1}$ & $\mathrm{a}_{1}$ & $\mathrm{~b}_{1}$ & $\mathrm{c}_{1}$ \\
2 & $\mathrm{a}_{2}$ & $\mathrm{a}_{2}$ & $\mathrm{~b}_{2}$ & $\mathrm{c}_{2}$ \\
3 & $\mathrm{a}_{3}$ & $\mathrm{a}_{3}$ & $\mathrm{~b}_{3}$ & $\mathrm{c}_{3}$ \\
4 & $\mathrm{a}_{4}$ & $\mathrm{a}_{4}$ & $\mathrm{~b}_{4}$ & $\mathrm{c}_{4}$ \\
\hline
\end{tabular}

\section{RESULTADOS}

\subsection{En la materia prima}

Se retiró el hueso de las aceitunas negras fermentadas y se pesó la pulpa, determinándose el $\mathrm{pH}$, la humedad y el rendimiento de pulpa de aceituna. Los resultados fueron los indicados en la Tabla $\mathrm{N}^{\circ} 02$.

Tabla $\mathrm{N}^{\circ} 02$. Características de la aceituna negra

\begin{tabular}{lc}
\hline \multicolumn{1}{c}{ Característica } & Contenido \\
\hline Humedad & $78,2 \%$ \\
$\mathrm{pH}$ & $4,4 \%$ \\
Rendimiento (pulpa/aceituna) & $58 \%$ \\
\hline
\end{tabular}

\subsection{Textura de la pasta de aceituna}

La Tabla $\mathrm{N}^{\circ} 03$, muestra los resultados de la evaluación sensorial de la pasta de aceituna negra, sin adición de hidrocoloide y con adición de hidrocoloide a concentraciones de $0 ; 0,5 ; 1$ y $1,5 \%$ respectivamente.

Tabla $N^{\circ} 03$. Textura de la pasta de aceituna

Prueba Concentración del hidrocoloide

\begin{tabular}{ccccc} 
& $\mathbf{0} \%$ & $\mathbf{0 , 5} \%$ & $\mathbf{1} \%$ & $\mathbf{1 , 5} \%$ \\
\hline 1 & 5 & 5 & 6 & 7 \\
2 & 5 & 6 & 7 & 5 \\
3 & 5 & 5 & 6 & 4 \\
4 & 6 & 6 & 5 & 6 \\
\hline
\end{tabular}

\subsection{Sensación en boca}

En la Tabla $\mathrm{N}^{\circ} 04$, se observa los resultados de la sensación en boca de la pasta de aceituna.

Tabla $N^{\circ} 04$. Sensación en boca de la pasta de aceituna

Prueba Concentración del hidrocoloide

\begin{tabular}{ccccc} 
& $\mathbf{0} \%$ & $\mathbf{0 , 5} \%$ & $\mathbf{1 \%}$ & $\mathbf{1 , 5 \%}$ \\
\hline 1 & 6 & 4 & 5 & 6 \\
2 & 5 & 6 & 4 & 5 \\
3 & 5 & 5 & 6 & 6 \\
4 & 3 & 3 & 5 & 4 \\
\hline
\end{tabular}




\subsection{Sinéresis}

En la Tabla $\mathrm{N}^{\circ} 05$, observamos los resultados de la exudación de líquido (sinéresis) en la pasta de aceituna elaborada.

Tabla $N^{\circ} 05$. Sinéresis en la pasta de aceituna

\begin{tabular}{ccccc}
\hline Prueba & \multicolumn{4}{c}{ Concentración del hidrocoloide } \\
& $\mathbf{0} \%$ & $\mathbf{0 , 5} \%$ & $\mathbf{1} \%$ & $\mathbf{1 , 5 \%}$ \\
\hline 1 & 1 & 3 & 3 & 5 \\
2 & 2 & 3 & 5 & 6 \\
3 & 1 & 2 & 5 & 6 \\
4 & 2 & 4 & 5 & 3 \\
\hline
\end{tabular}

\subsection{Aspecto general}

En el Tabla $\mathrm{N}^{\circ} 06$ se observa el aspecto general presentado por las muestras de pasta de aceituna negra.

Tabla $N^{\circ} 06$. Aspecto general de la pasta de aceituna

Prueba Concentración del hidrocoloide

\begin{tabular}{ccccc} 
& $\mathbf{0} \%$ & $\mathbf{0 , 5} \%$ & $\mathbf{1} \%$ & $\mathbf{1 , 5} \%$ \\
\hline 1 & 1 & 3 & 5 & 6 \\
2 & 1 & 2 & 4 & 6 \\
3 & 1 & 3 & 3 & 6 \\
4 & 2 & 3 & 5 & 4 \\
\hline
\end{tabular}

\subsection{Flujo del proceso}

La Figura $\mathrm{N}^{\circ} 03$, muestra las operaciones realizadas para la elaboración de la pasta de aceituna negra.

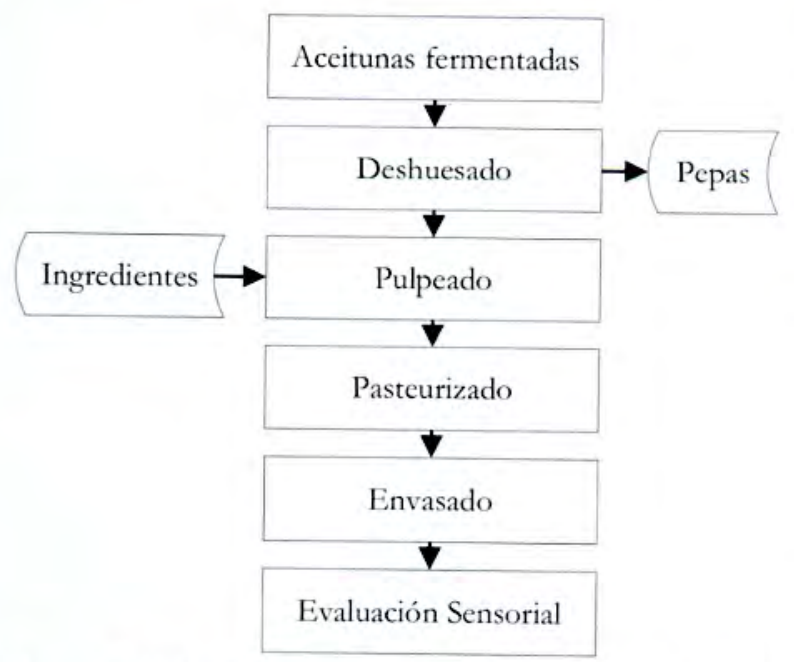

Figura $\mathrm{N}^{\circ} 03$. Flujo de proceso realizado en la elaboración de pasta de aceituna

\subsection{Análisis estadístico}

Las tablas $\mathrm{N}^{\circ} 07,08,09$ y 10 muestran el análisis de varianza de las muestras de pasta de aceituna, en lo referido a la textura, sensación en boca, sinéresis y aspecto general respectivamente.
Tabla $\mathrm{N}^{\circ} 07$. ANVA para la textura de la pasta de aceituna

\begin{tabular}{lcccc}
\hline \multicolumn{1}{c}{ Fuente de Variación } & g.I. & SC & CM & F \\
\hline Tratamiento (Muestras) & 3 & 1,1875000 & 0,3958333 & 0,504 \\
Prucba & 3 & 1,6875000 & 0,5625000 & 0,717 \\
Error & 9 & 7,0625000 & 0,7847222 & \\
Total & 15 & 9,9375000 & & \\
\hline
\end{tabular}

Tabla $\mathrm{N}^{\circ} 08$. ANVA para la sensación en boca de la pasta de aceituna

\begin{tabular}{lcccc}
\hline \multicolumn{1}{c}{ Fuente de Variación } & g.l. & SC & CM & F \\
\hline Tratamiento (Muestras) & 3 & 1,2500000 & 0,4166667 & 0,517 \\
Prueba & 3 & 7,2500000 & 2,4166667 & 3,000 \\
Error & 9 & 7,2500000 & 0,8055556 & \\
Total & 15 & 15,7500000 & & \\
\hline
\end{tabular}

Tabla $\mathrm{N}^{\circ} 09$. ANVA para la sinéresis de la pasta de aceituna

\begin{tabular}{lcccc}
\hline \multicolumn{1}{c}{ Fuente de Variación } & g.l. & SC & CM & F \\
\hline Tratamiento (Muestras) & 3 & 30,0000000 & 10,0000000 & 9,000 \\
Prueba & 3 & 2,0000000 & 0,6666667 & 0,600 \\
Error & 9 & 10,0000000 & 1,1111111 & \\
Total & 15 & 42,0000000 & & \\
\hline
\end{tabular}

Tabla $\mathrm{N}^{\circ} 10$. ANVA para el aspecto general de la pasta de aceituna

\begin{tabular}{lcccc}
\hline \multicolumn{1}{c}{ Fuente de Variación } & g.I. & SC & CM & F \\
\hline Tratamiento (Muestras) & 3 & 40,6875000 & 13,5625000 & 18,600 \\
Prucba & 3 & 0,6875000 & 0,2291667 & 0,314 \\
Error & 9 & 6,5625000 & 0,7291667 & \\
Total & 15 & 47,9375000 & & \\
\hline
\end{tabular}

\section{ANÁLISIS Y DISCUSIÓN DE RESULTADOS}

Del análisis de varianza (ANVA) con un valor de F teórico, con 3 grados de libertad y 15 grados de libertad de 3,29 ; y valores de $F$ calculados de 0,$504 ; 0,517 ; 9,0$ y 18,6 para la textura, sensación en boca, sinéresis y aspecto general respectivamente, se deduce lo siguiente:

- La textura de la pasta de aceituna, entre los tratamientos con hidrocoloide y sin hidrocoloide, no presentan diferencias significativas con $95 \%$ de confianza.

- La sensación en boca de la pasta de aceituna, entre los tratamientos sin hidrocoloide y con hidrocoloide, no presentan diferencias significativas con $95 \%$ de confianza.

- Hay diferencia significativa entre los tratamientos realizados con hidrocoloide y sin hidrocoloide, con $95 \%$ de confianza, referido a la sinéresis y al aspecto general de la pasta de aceituna.

Realizando la prueba de Tukey, con 95\% de confianza, se determinó que no existe diferencia significativa para los tratamientos con 1,0 y $1,5 \%$ de hidrocoloide en la pasta de aceituna.

\section{CONCLUSIONES}

Se determinó que el uso de hidrocoloide en la pasta 
de aceituna tiene efecto en evitar la sinéresis manteniendo un mejor aspecto general.

La concentración de hidrocoloide a evita la sinéresis y da mejor aspecto general a la pasta de aceituna está a concentraciones de 1 a 1,5\%.

Se determinó que el principal aporte a la calidad de la pasta de aceituna negra es en evitar la sinéresis y mejora del aspecto general.

\section{RECOMENDACIONES}

Se recomienda determinar la mejor concentración del uso del hidrocoloide entre los valores de 1 a $1,5 \%$ de concentración en la pasta de aceituna negra.

Se recomienda evaluar la influencia de otras variables en la estabilidad de la pasta de aceituna como por ejemplo: tratamiento térmico, tiempo de conservación, temperatura de conservación.

\section{REFERENCIAS BIBLIOGRÁFICAS}

Badui S. (1993). Química de los Alimentos. México D.F, México. Editorial Alambra Mexicana.

Bailley A. (1984). Aceites y grasas industriales. Barcelona España. Editorial RevertéS.A.

Bello Gutierrez José. (2000). Ciencia Bromatológica: Principios Generales de los Alimentos. Madrid España. Ediciones Díaz de Santos.
Cabello I., (2009). Monografía para el cultivo de la tara Caesalpinia Spinosa. Lima Perú.

Cubero N., Monferrer A., Villalta J. (2002). Aditivos Alimentarios. Madrid España. Mundi-Prensa Libros.

Desrosier N. (1974). Tecnología de la conservación de Alimentos, Editorial Continental, México

Hashiguchi P. (2005). Tesis: Formulación de un producto moldeado a base de pasta de aceitunas: Aceitunas Duquesa. Para optar el título de Ingeniero de Alimentos Universidad de Chile. Facultad de Ciencias Químicas y Farmacéuticas. Santiago Chile.

Instituto de la grasa y sus derivados. (1985). Biotecnología de la aceituna de mesa. Sevilla España.

Instituto Interamericano de Cooperación para la Agricultura (IICA). 1989. Serie de publicaciones misceláneas. Estudio sobre, producción e industrialización de la aceituna y subproductos en Tacna. Oficina del IICA. Lima-Perú.

Loussert y Browsse (1980). El olivo. Madrid España. Ediciones Mundi Prensa.

Mora F., Barraza G., Obregón J. Sinéresis, características reológicas y consistencia sensorial de salsa de alcachofa (Cynara scolymus L.). Scientia Agropecuaria. 4(2013), 163172.

Schwartz M., Quitral V., Daccarett C. y Callejas J. (2000). Desarrollo de una pasta untable de aceituna variedad Sevillana. Grasas y aceites. 60(5), 451-457.
Correspondencia:

Marcial Castillo Cohaila: mcastilloc@unjbg.edu.pe
Fecha de Recepción: 06/05/2014

Fecha de Aceptación: 18/06/2014 